\title{
Search for additional neutral MSSM Higgs bosons in the di-tau final state
}

\author{
Markus Spanring ${ }^{* \dagger}$ \\ HEPHY, Austrian Academy of Sciences \\ E-mail: markus.spanring@cern.ch
}

\begin{abstract}
A search for additional neutral Higgs bosons in the $\tau \tau$ final state is presented. The search is performed in the context of the minimal supersymmetric extension of the standard model (MSSM), using data from proton-proton collision collected with the CMS detector in 2016 at a center-ofmass energy of $13 \mathrm{TeV}$. The collected data corresponds to an integrated luminosity of $35.9 \mathrm{fb}^{-1}$. No significant deviation above the expected background is observed. Model-independent limits at 95\% confidence level are set on the product of the branching fraction for the decay into $\tau$ leptons and the cross section of the production mode.
\end{abstract}

An Alpine LHC Physics Summit (ALPS2018)

15-20 April, 2018

Obergurgl, Austria

* Speaker.

${ }^{\dagger}$ on behalf of the CMS Collaboration. 


\section{Introduction}

The discovery of a Higgs boson by the ATLAS and CMS experiments in 2012 [1-3] provided evidence that spontaneous symmetry breaking may indeed be realized in nature as proposed by the Brout-Englert-Higgs mechanism. However, the standard model (SM) is known not to be complete and a number of favoured beyond the standard model (BSM) theories include an extended Higgs sector.

The minimal supersymmetric extension of the standard model (MSSM) contains two scalar doublets that result in five physical Higgs bosons: two charged Higgs bosons $\mathrm{H}^{ \pm}$, two neutral scalar Higgs bosons $\mathrm{h}$ and $\mathrm{H}$ and one neutral pseudoscalar $\mathrm{A}$. It is conventional to make use of the notation $\phi$ to represent any one of the three neutral Higgs bosons, $\phi=h, H, A$.

At tree-level in the MSSM, the masses of these five Higgs bosons and their mixing can be expressed in terms of the gauge boson masses and two additional parameters, which can be chosen as the mass of the pseudoscalar $\mathrm{A}$ and the ratio of the vacuum expectation values of the neutral components of the two Higgs doublets

$$
\tan \beta=\frac{\left\langle H_{u}^{0}\right\rangle}{\left\langle H_{d}^{0}\right\rangle}=\frac{v_{u}}{v_{d}} .
$$

At leading-order (LO), the coupling to down-type fermions is enhanced by $\tan \beta$ with respect to the expectation for an SM Higgs boson of the same mass. This leads to two effects: enhanced rate of decays into $\tau$ leptons, and a second dominant production mode, $\mathrm{b}$ associated production.

The presented results of a search for additional heavy Higgs bosons in the context of the MSSM [4] are based on the $2016 \mathrm{pp}$ collision data, taken at a center-of-mass energy of $13 \mathrm{TeV}$ by the CMS experiment, and corresponding to an integrated luminosity of $35.9 \mathrm{fb}^{-1}$. Four different $\tau \tau$ signatures are studied, $\mathrm{e} \mu, \mathrm{e} \tau_{\mathrm{h}}, \mu \tau_{h}$ and $\tau_{h} \tau_{h}$, where $\tau_{h}$ denotes a hadronically decaying $\tau$. For this analysis the most significant backgrounds are estimated from data, by using new techniques with respect to the previous publication by CMS [5].

The results are interpreted in the context of the MSSM with different benchmark scenarios and also in a model independent way, in terms of upper limits on the cross section times branching fraction $\sigma \mathscr{B}(\phi \rightarrow \tau \tau)$.

\section{Analysis strategy}

\subsection{Categorization}

In this analysis the four most sensitive final states, e $\mu$, e $\tau_{\mathrm{h}}, \mu \tau_{h}$, and $\tau_{h} \tau_{h}$, are exploited. To further increase the sensitivity all selected events are further categorized: events with at least one jet passing the $b$ tagging requirement are combined into a global b-tag category. This category is designed to target the production of the Higgs boson in association with $b$ quarks. All other events are added to a global no b-tag category. 
In the $\mathrm{e} \mu$ final state each event category is further split into three subcategories based on the quantity $D_{\zeta}[6]$ defined as

$$
D_{\zeta}=p_{\zeta}^{\text {miss }}-1.85 p_{\zeta}^{\text {vis }} ; \quad p_{\zeta}^{\text {miss }}=\vec{p}_{\mathrm{T}}^{\text {miss }} \hat{\zeta} ; \quad p_{\zeta}^{\text {vis }}=\left(\vec{p}_{\mathrm{T}}^{\mathrm{e}}+\vec{p}_{\mathrm{T}}^{\mu}\right) \hat{\zeta}
$$

where $\vec{p}_{\mathrm{T}}^{\mathrm{e}(\mu)}$ corresponds to the transverse momentum vector of the electron (muon) and $\hat{\zeta}$ to the bisectional direction between the electron and the muon in the transverse plane. The categories are defined as low- $D_{\zeta}$, medium- $D_{\zeta}$ and high- $D_{\zeta}$ (Fig. 1 left). The expected signal, for all masses tested, is mostly located in the medium $-D_{\zeta}$ subcategory.

In the $\mathrm{e} \tau_{\mathrm{h}}\left(\mu \tau_{h}\right)$ final state each global event category is further split into two subcategories based on the transverse mass of the electron (muon):

$$
m_{\mathrm{T}}^{e(\mu)}=\sqrt{2 p_{\mathrm{T}}^{e(\mu)} p_{\mathrm{T}}^{\mathrm{miss}} \cdot(1-\cos \Delta \phi)}
$$

In Eq. (2.2) $p_{\mathrm{T}}^{e(\mu)}$ refers to the $p_{\mathrm{T}}$ of the electron (muon) and $\Delta \phi$ to the difference in the azimuthal angle between the electron (muon) and $p_{\mathrm{T}}^{\text {miss }}$. The categories are defined as tight- $m_{\mathrm{T}}$ and loose- $m_{\mathrm{T}}$ (Fig. 1 right) where the bulk of the signal events, particularly for the low-mass hypotheses, lie in the tight- $m_{\mathrm{T}}$ subcategory. The loose- $m_{\mathrm{T}}$ category has been added to increase the signal acceptance for mass hypotheses of $m_{\mathrm{A}, \mathrm{H}}>700 \mathrm{GeV}$.

In combination this leads to 16 event categories entering the statistical analysis, complemented by three background control regions, used for normalization of dominant backgrounds estimated from simulation.
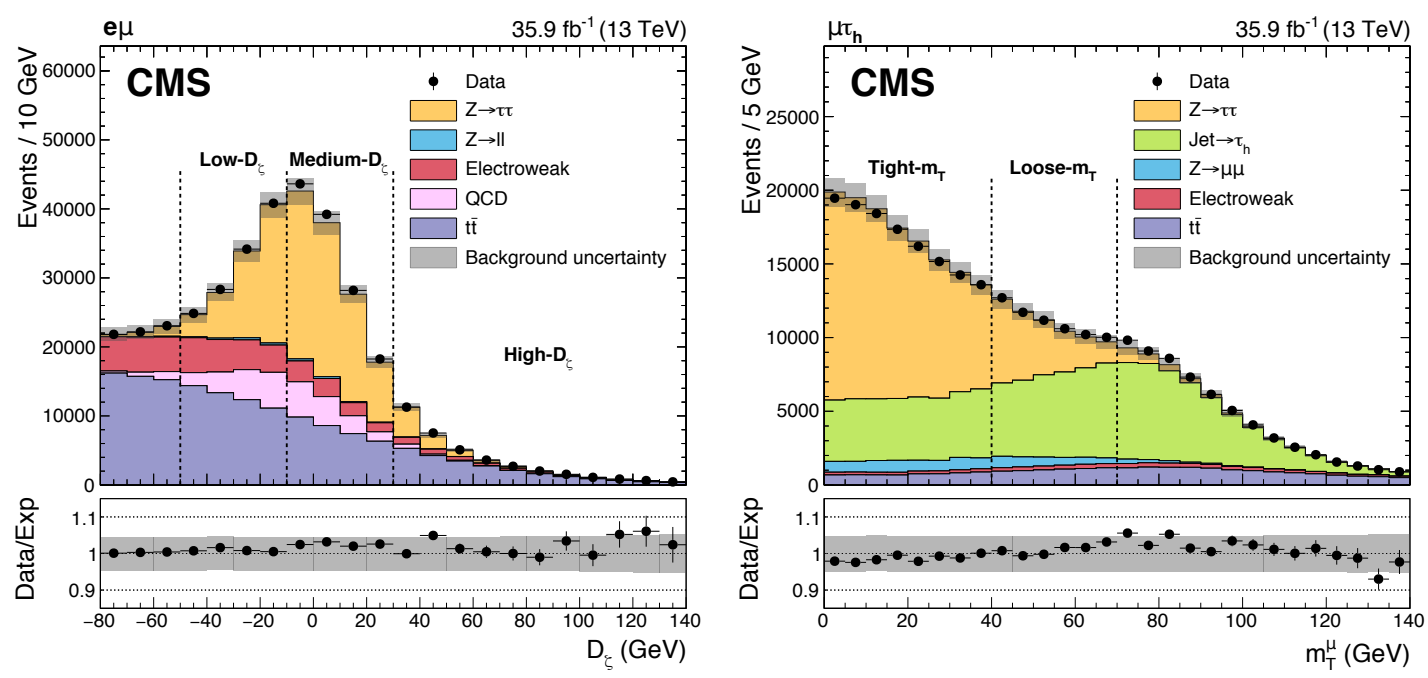

Figure 1: Observed and expected distributions of (left) $D_{\zeta}$ in the e $\mu$ final state and (right) $m_{\mathrm{T}}^{\mu}$ in the $\mu \tau_{h}$ final state. The dashed vertical lines indicate the definition of the subcategories in each final state. The label "jet $\rightarrow \tau_{\mathrm{h}}$ " indicates events with jets misidentified as hadronic $\tau$ lepton decays, e.g. $\mathrm{W}+$ jets events, which are estimated from data as described in Section 2.2 [4]. 


\subsection{Background estimation}

A large fraction of the backgrounds in the final states containing a hadronically decaying $\tau$ lepton, $\mathrm{e} \tau_{\mathrm{h}}, \mu \tau_{h}$ and $\tau_{h} \tau_{h}$, can be attributed to jets misidentified as hadronic $\tau$ lepton decays. To estimate these backgrounds, shape and normalization are taken from control regions in data using the "fake factor" method [7]. The number of events for a certain background $i$ due to a misidentified jet is estimated from a region that differs from the signal region (SR) only by a modified $\tau_{h}$ identification requirement, referred to as the application region (AR). Here, the $\tau_{h}$ identification is required to fulfill the VeryLoose but not Tight (Medium) working point of the discriminant in the $\mathrm{e} \tau_{\mathrm{h}} / \mu \tau_{h}$ $\left(\tau_{h} \tau_{h}\right)$ final state.

To get an estimate for the number of events from background $i$ due to jet $\rightarrow \tau_{h}$ misidentification in the SR the number of events is multiplied by the ratio

$$
F_{F}^{i}=\frac{N_{\text {pass }}}{N_{\text {fail }}},
$$

where $N_{\text {pass }}$ corresponds to the number of events that fulfill the Tight/Medium working point and $N_{\text {fail }}$ to the number of events that fulfill the VeryLoose but not the Tight/Medium working point of the identification discriminant. The ratio in Eq. (2.3) is obtained in a determination region $\left(\mathrm{DR}_{\mathrm{i}}\right)$, which is orthogonal to the $\mathrm{AR}$ and $\mathrm{SR}$, and dominated by background $i$.

The underlying assumption in this method is that the ratio of the number of events from background $i$ in the $\mathrm{SR}$ to the number of events in the AR is equal to the ratio $N_{\text {pass }} / N_{\text {fail }}$ in the respective determination region $\mathrm{DR}_{\mathrm{i}}$.

From the individually determined $F_{F}^{i}$ a weighted factor is obtained on an event-by-event basis from

$$
F_{F}=\sum_{i} w_{i} F_{F}^{i}, \quad w_{i}=\frac{N_{A R}^{i}}{\sum_{j} N_{A R}^{j}}, \quad \text { with } \quad i, j \in\{\mathrm{QCD}, \mathrm{W}+\text { jets, }, \overline{\mathrm{t}}\},
$$

where $N_{A R}^{i}$ corresponds to the expected number of events for background $i$ in the AR. The $F_{F}$ is then applied to all events in the AR to obtain an estimate for the normalization and shape of the sum of QCD multijet, $\mathrm{W}+\mathrm{jets}$, and $t \bar{t}$ events due to the jet $\rightarrow \tau_{h}$ misidentification. The principal method is outlined in Fig. 2.

In the $\mathrm{e} \mu$ final state the background from QCD multijet events is estimated from an AR fulfilling the same selection requirements as the SR, but the charges of the leptons are required to be of the same sign. For the transition from the same charge to the opposite charge phase space extrapolation factors are derived in a DR region without event categorization, in which the isolation requirements of the leptons are chosen to be orthogonal to the SR.

All other backgrounds, apart from the ones described above, are estimates from simulation where the dominant backgrounds with genuine hadronic $\tau$ leptons are normalized in control regions. 


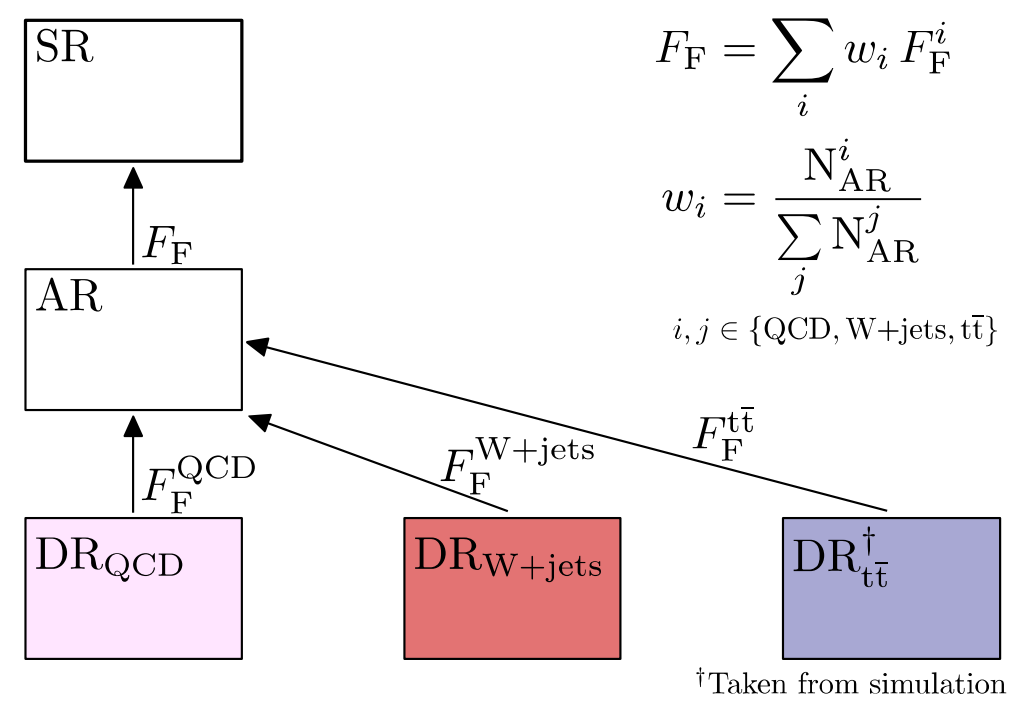

Figure 2: Schematic of the determination and application of the $F_{F}^{i}$ and $F_{F}$ for the estimation of the background from QCD multijet, $\mathrm{W}+\mathrm{jets}$, and $t \bar{t}$ events due to the misidentification of jets as hadronic $\tau$ lepton decays [4].

\section{Results}

The final discriminating variable used to search for signal is the total transverse mass $m_{\mathrm{T}}^{\text {tot }}$, which is defined as

$$
m_{\mathrm{T}}^{\text {tot }}=\sqrt{m_{\mathrm{T}}^{2}\left(p_{T}^{\tau_{1}}, p_{T}^{\tau_{2}}\right)+m_{\mathrm{T}}^{2}\left(p_{T}^{\tau_{1}}, p_{T}^{\text {miss }}\right)+m_{\mathrm{T}}^{2}\left(p_{T}^{\tau_{2}}, p_{T}^{\text {miss }}\right)},
$$

where the pair $\left(\tau_{1}, \tau_{2}\right)$ can be $(\mathrm{e}, \mu),\left(\mathrm{e}, \tau_{\mathrm{h}}\right),\left(\mu, \tau_{h}\right)$ or $\left(\tau_{h}, \tau_{h}\right)$ and the transverse mass, $m_{\mathrm{T}}$, between two objects with transverse momenta $p_{\mathrm{T}}$ and $p_{\mathrm{T}}^{\prime}$, and relative difference $\Delta \phi$ in the azimuthal angle is given by:

$$
m_{\mathrm{T}}=\sqrt{2 p_{\mathrm{T}} p_{\mathrm{T}}^{\prime} \cdot(1-\cos \Delta \phi)} .
$$

The input distributions to the statistical inference of the signal in a subset of the most sensitive event subcategories per final state are shown in Fig. 3.

In the first interpretation of the data 95\% confidence level (CL) upper limits are set on the product of the branching fraction for the decay into $\tau$ leptons and the cross section for the production of a single narrow width resonance, $\phi$, via gluon fusion or in association with b quarks. In Fig. 4 these limits are shown as a function of $m_{\phi}$. The expectation for an SM Higgs boson at $125 \mathrm{GeV}$ is taken into account in the SM backgrounds. For the production via gluon fusion the expected limits range between $18 \mathrm{pb}$ at $m_{\phi}=90 \mathrm{GeV}$ and $3.5 \mathrm{fb}$ at $m_{\phi}=3.2 \mathrm{TeV}$. For the production in association with 

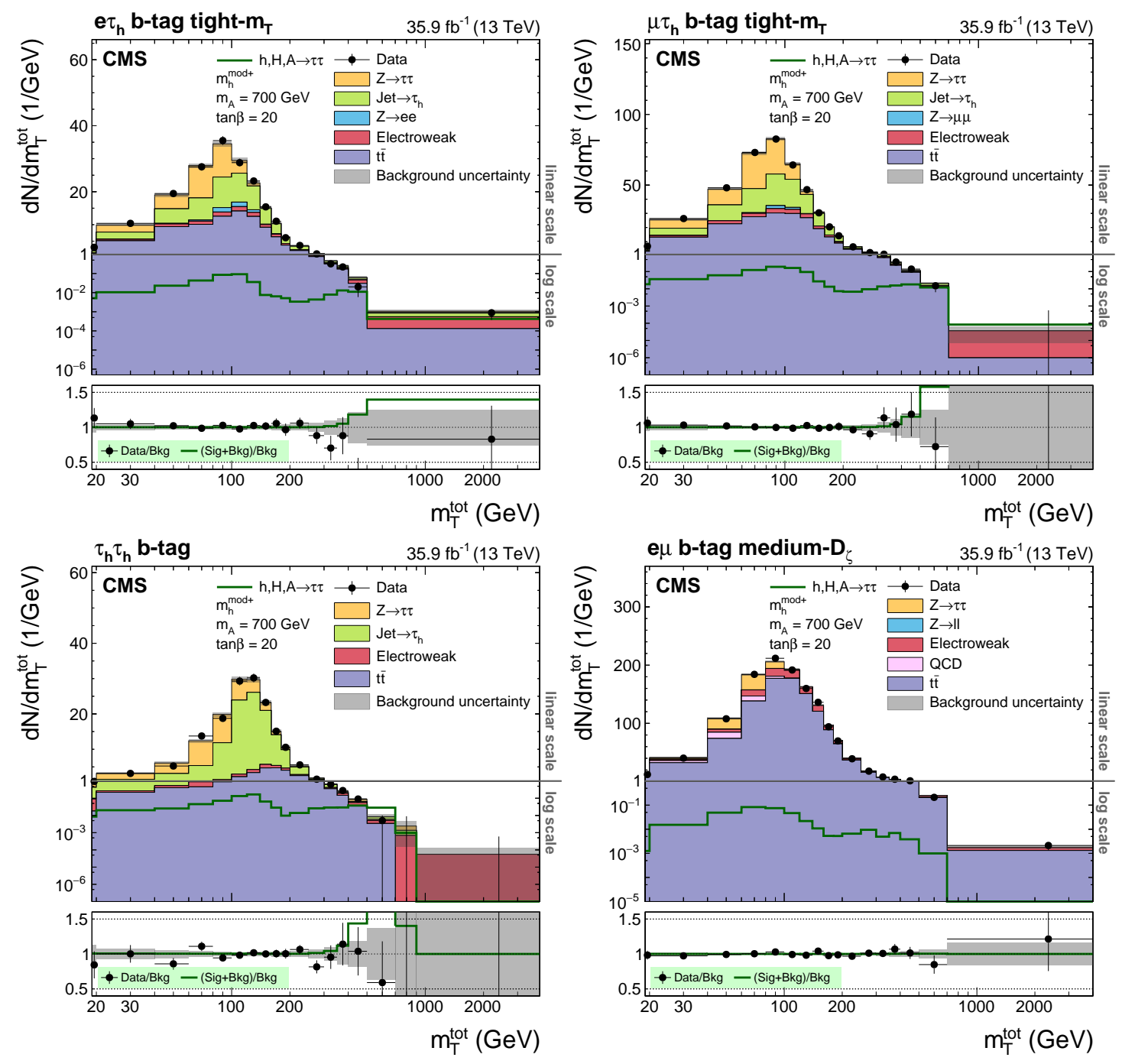

Figure 3: Distribution of $m_{\mathrm{T}}^{\text {tot }}$ for the b-tag categories in the e $\tau_{\mathrm{h}}$ (upper left), $\mu \tau_{h}$ (upper right), $\tau_{h} \tau_{h}$ (lower left) and $\mathrm{e} \mu$ (lower right) final states. For the $\mathrm{e} \tau_{\mathrm{h}}$ and $\mu \tau_{h}$ final state the most sensitive tight- $m_{\mathrm{T}}$ event subcategory is shown. For the e $\mu$ final state the medium- $D_{\zeta}$ event subcategory is shown. The gray horizontal line in the upper panel of each subfigure indicates the change from logarithmic to linear scale on the vertical axis [4].

b quarks they range between $15 \mathrm{pb}$ at $m_{\phi}=90 \mathrm{GeV}$ and $2.5 \mathrm{fb}$ at $m_{\phi}=3.2 \mathrm{TeV}$. In both cases the excluded cross section falls with increasing mass, before becoming constant at around $1 \mathrm{TeV}$. No significant deviation from the expectation is observed.

In the second interpretation of the data, exclusion contours in the $m_{\mathrm{A}}-\tan \beta$ plane are determined for two representative benchmark scenarios of the MSSM, the $m_{\mathrm{H}}^{\bmod +}$ and the hMSSM. In Fig. 5 exclusion contours extend down to values of $\tan \beta \approx 6$ for values of $m_{\mathrm{A}} \lesssim 250 \mathrm{GeV}$. For the $m_{\mathrm{H}}^{\bmod +}$ scenario those parts of the parameter space in which $m_{\mathrm{h}}$ deviates by more than $\pm 3 \mathrm{GeV}$ from the mass of the observed Higgs boson at $125 \mathrm{GeV}$ are indicated by a red hatched area. Over the whole 
mass range the observed exclusion contours follow the expectation with the largest deviations still contained in the $95 \%$ confidence interval for the variation of the expected exclusion.
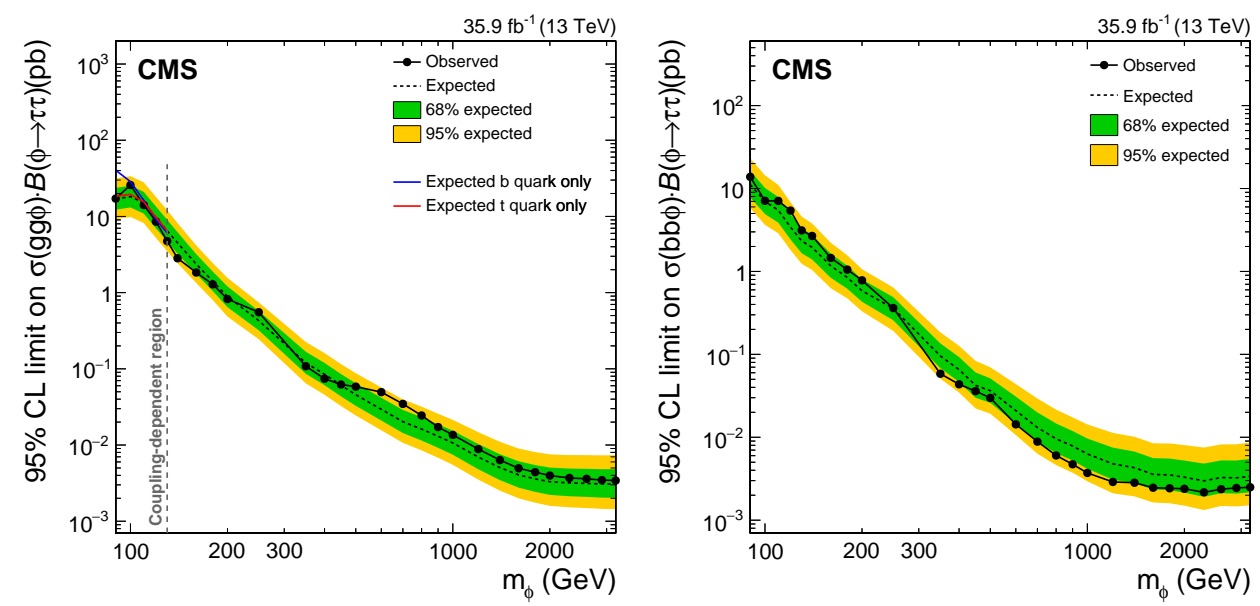

Figure 4: Expected and observed 95\% CL upper limits for the production of a single narrow resonance, $\phi$, with a mass between $90 \mathrm{GeV}$ and $3.2 \mathrm{TeV}$ in the $\tau \tau$ final state (left) for the production via gluon fusion $(\operatorname{gg} \phi)$ and (right) in association with b quarks (bb $\phi)$ [4].
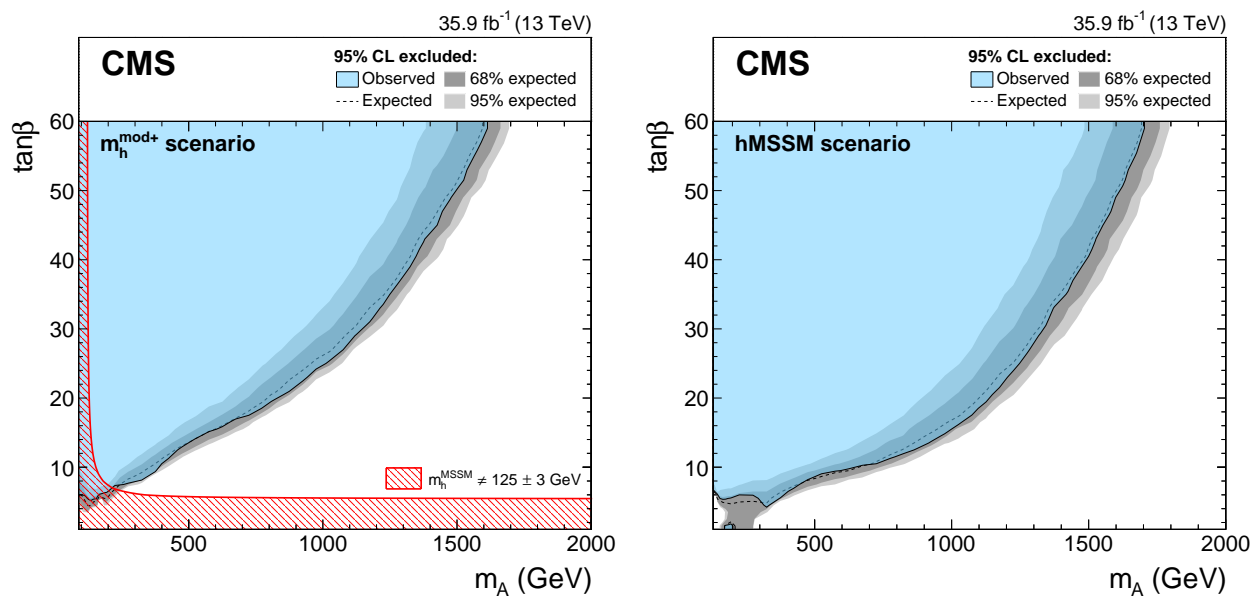

Figure 5: Expected and observed $95 \% \mathrm{CL}$ exclusion contour (left) in the MSSM $m_{\mathrm{H}}^{\bmod +}$ and (right) in the hMSSM scenarios. The expected median is shown as a dashed black line. The dark and bright gray bands indicate the 68 and $95 \%$ confidence intervals for the variation of the expected exclusion. The observed exclusion contour is indicated by the colored blue area. For the $m_{\mathrm{H}}^{\bmod +}$ scenario, those parts of the parameter space, where $m_{\mathrm{h}}$ deviates by more than $\pm 3 \mathrm{GeV}$ from the mass of the observed Higgs boson at $125 \mathrm{GeV}$ are indicated by a red hatched area [4]. 


\section{Summary}

A search for neutral Higgs bosons in the context of the minimal supersymmetric extension of the standard model decaying to $\tau$-lepton pairs has been presented. The dataset corresponds to an integrated luminosity of $35.9 \mathrm{fb}^{-1}$ at $13 \mathrm{TeV}$. This search has been presented in the most sensitive final states of the $\tau \tau$ pair e $\mu$, e $\tau_{\mathrm{h}}, \mu \tau_{h}$ and $\tau_{h} \tau_{h}$, where $\tau_{h}$ denotes a hadronically decaying $\tau$ lepton. No excess has been observed. Model independent limits at $95 \%$ confidence level have been set for the production of a single narrow resonance decaying into a pair of $\tau$ leptons. For the production via gluon fusion the expected limits range between $18 \mathrm{pb}$ at $m_{\phi}=90 \mathrm{GeV}$ and $3.5 \mathrm{fb}$ at $m_{\phi}=3.2 \mathrm{TeV}$. For the production in association with $\mathrm{b}$ quarks they range between $15 \mathrm{pb}$ at $m_{\phi}=90 \mathrm{GeV}$ and $2.5 \mathrm{fb}$ at $m_{\phi}=3.2$. Finally $95 \%$ confidence level exclusion contours have been provided for the $m_{\mathrm{H}}^{\bmod +}$ and the hMSSM scenarios. These extend down to values of $\tan \beta \approx 6$ for values of $m_{A} \lesssim 250 \mathrm{GeV}$ and reach $1.6 \mathrm{TeV}$ for $\tan \beta=60$.

\section{References}

[1] ATLAS Collaboration, Observation of a new particle in the search for the Standard Model Higgs boson with the ATLAS detector at the LHC, Phys. Lett. B716 (2012) 1 [1207. 7214].

[2] CMS Collaboration, Observation of a new boson at a mass of $125 \mathrm{GeV}$ with the CMS experiment at the LHC, Phys. Lett. B716 (2012) 30 [1207 . 7235].

[3] CMS Collaboration, Observation of a new boson with mass near $125 \mathrm{GeV}$ in pp collisions at $\sqrt{s}=7$ and 8 TeV, JHEP 06 (2013) 081 [1303.4571].

[4] CMS Collaboration, Search for additional neutral MSSM Higgs bosons in the $\tau \tau \tau$ final state in proton-proton collisions at $\sqrt{s}=13 \mathrm{TeV}, 1803.06553$.

[5] CMS Collaboration, Search for neutral MSSM Higgs bosons decaying to a pair of tau leptons in pp collisions, JHEP 10 (2014) 160 [1408.3316].

[6] CDF Collaboration, Search for neutral MSSM Higgs bosons decaying to tau pairs in p p $\bar{p}$ collisions at $\sqrt{s}=1.96$ TeV, Phys. Rev. Lett. 96 (2006) 011802 [hep-ex/ 0508051 ].

[7] CMS Collaboration, Measurement of the $\mathrm{Z} \gamma^{*} \rightarrow \tau \tau$ cross section in pp collisions at $\sqrt{s}=13 \mathrm{TeV}$ and validation of $\tau$ lepton analysis techniques, 1801.03535. 